\title{
Stereological determination of dry-snow parameters for discrete-scatterer microwave modeling
}

\author{
JIANCHENG SHI, \\ Center for Remote Sensing and Environmental Optics, University of California, Santa Barbara, CA 93106, U.S.A. \\ ROBERT E. DAVIS, \\ U.S. Army Cold Regions Research and Engineering Laboratory, Hanover, NH 03755-1290, U.S.A. \\ JEFF DOZIER \\ NASA Godddard Space Flight Center, Code 900, Greenbelt, MD 20771, U.S.A.
}

\begin{abstract}
Modeling microwave back-scattering and emission from snowpacks requires the knowledge of snowpack characteristics and their dynamics to select an appropriate model. Both theory and field data show that microwave backscattering coefficients and brightness temperatures are sensitive to parameters describing snow-microstructure. Stereological methods and other techniques can be applied to images of sections cut from undisturbed snow, and are used to obtain accurate and unbiased estimates of snow-microstructure parameters for discrete scatterer modeling. Assuming that the ice particle-size distribution can be characterized as a log-normal distribution function, we show that the parameters describing the distribution can be obtained from section images. The results show that, in addition to snow density and ice-particle size, the particle-size variation has great effect on dry-snow extinction properties. The optically equivalent ice-particle size for Rayleigh scattering in a snowpack with grain-size variations can be determined from the stereological measurements from snow sections.
\end{abstract}

\section{INTRODUGTION}

The main problem in estimating snow properties from remote-sensing data is understanding the links between the electromagnetic interactions in different parts of the spectrum and the physical properties of the snow. Both theory (e.g. Ding and Tsang, 1988; Tsang, 1992) and field measurements (Hüppe, 1986) of microwave signals from snowpacks show that the back-scattering coefficient and the microwave brightness temperature are sensitive to parameters describing snow microstructure. Repeatable and unbiased estimates of snow microstructure and grain-size indices can be obtained from stereological methods applied to images of sections cut from undisturbed snow.

Stereology is a statistically based method by which quantities measured directly from a two-dimensional plane intersecting a three-dimensional body are used to obtain average values for the three-dimensional structure of the body (Underwood, 1970). In practice, this involves filling the pore space of snow specimens collected from the field, then preparing polished sections from which images are constructed. Recent snow studies on stereological methods applied to snow (Davis and Dozier, 1989) show that the first-moment statistical parameters - mean values, such as ice-profile size, effective number of particles per unit volume and structure anisotropy, can be obtained from the measurements of the plane sections. For example, a grain-size index equivalent to the sphere with equal surface-to-volume ratio of the ice matrix has been shown to be very close to the optical grain-size in the visible and near-infrared spectrum (Dozier, 1989). These measurements can be made without assuming specific shapes.

In situations where Rayleigh scattering dominates, such as the microwave spectral region, larger particles play a more important role than smaller ones in the interactions between the electromagnetic waves and the ice particles. Because ice particle-size variations are always expected in natural snowpacks, significantly different extinction properties will be observed. Therefore, it is necessary to evaluate the effects of variations in ice-particle size on snow-extinction properties. In this work, we describe the stereological measurements required to estimate the ice particle-size distribution and its parameters. The effects of ice-particle variation on dry-snow extinction are evaluated for discrete scatterer modeling and Rayleigh scattering. We then demonstrate a particle-size measurement to determine the optically equivalent grain-size for a snowpack. 


\section{STEREOLOGICAL DETERMINATION OF DRY- SNOW PROPERTIES}

The snow parameters required by discrete-scatter models include the mean ice-particle size and the variance of particle sizes or size distribution. The volume fraction of ice, dielectric property of ice, and shape and orientation of particles are required, if they cannot be described by spheres. All of the above dry-snow properties can be directly measured from plane sections.

Measurements from a section plane are generally based upon three counting methods: point, line and area. The ice-volume fraction $v_{\mathrm{t}}$ obtained from the measuring one of the point fraction $P_{\mathrm{P}}$, lineal fraction $L_{\mathrm{L}}$ and area fraction $A_{\mathrm{A}}$.

$$
v_{\mathrm{t}}=P_{\mathrm{P}}+L_{\mathrm{L}}=A_{\mathrm{A}}
$$

where, for example, the point fraction is the ratio of pixels falling on ice to pixels falling on pore space in an image. Other important parameters needed for ice-grain indices are the surface density $S_{\mathrm{V}}$, intercept density $N_{\mathrm{L}}$ and mean intercept length $\bar{L}$, as described by Underwood (1970), and applied to microwave remote sensing of snow by Davis and Dozier (1989).

Small-particle systems can be commonly characterized by log-normal size distributions. This has been measured for falling snow in the absence of large aggregates by Koh (1984) and is suggested from the results of Wakahama (1960) and Colbeck (1986) for metamorphosed snow. The log-normal distribution function can be uniquely defined by $D_{\mathrm{g}}$ (the geometric mean diameter) and $\sigma_{\mathrm{g}}$ (the geometric standard deviation of diameters). Underwood (1970) showed that for lognormally distributed particles, $D_{\mathrm{g}}$ and $\sigma_{\mathrm{g}}$ can be estimated by simple stereological counting without knowledge or specification of the distribution curve.

$$
\log ^{2} \sigma_{\mathrm{g}}=\log \left(\frac{P_{\mathrm{P}} N_{\mathrm{A}}}{N_{\mathrm{L}}^{2}}\right)+\log K_{\sigma},
$$

and

$$
\log D_{\mathrm{g}}{ }^{2}=\log \left(\frac{N_{\mathrm{L}}{ }^{8}}{N_{\mathrm{A}}{ }^{5} P_{\mathrm{P}}{ }^{3}}\right)+\log K_{\mathrm{D}}
$$

where $K_{\mathrm{D}}$ and $K_{\sigma}$ are two shape-factor terms, which can be determined by

$$
\begin{aligned}
& K_{\mathrm{D}}=\frac{256 \alpha^{5} \gamma^{3}}{\beta^{8}} \\
& K_{\sigma}=\frac{\beta^{2}}{4 \alpha \gamma}
\end{aligned}
$$

where $\alpha, \beta$ and $\gamma$ are the shape-sensitivity factors.

An expression for the number of particles in a containing volume $N_{\mathrm{V}}$ has also been given by DeHoff (1965) for a size distribution of particles, which all have the same shape

$$
N_{\mathrm{V}}=\frac{N_{\mathrm{A}}}{\alpha \bar{L}}=\frac{S_{\mathrm{V}}}{\beta \bar{L}^{2}}=\frac{V_{\mathrm{V}}}{\gamma \bar{L}^{3}}
$$

where $\bar{L}, \bar{L}^{2}$ and $\bar{L}^{3}$ are related to the mean of the first, second and third moments of the intersection lengths, respectively. Using Equations (1)-(9), we find

$$
\log ^{2} \sigma_{\mathrm{g}}=\log \left(\frac{\bar{L} \bar{L}^{3}}{{\overline{L^{2}}}^{2}}\right)
$$

and

$$
D_{\mathrm{g}} \frac{=\bar{L}^{4}}{\bar{L}^{2} \bar{L}^{3} \sqrt{\left(\bar{L} \bar{L}^{3}\right)}}
$$

Therefore, the two parameters $D_{\mathrm{g}}$ and $\sigma_{\mathrm{g}}$ can also be obtained without knowledge of the statistical shape. The only assumption made here is that all ice particles have the same shape. In terms of the particle diameters $D$, the log-normal distribution is given by

$$
y=\frac{1}{\log \sigma_{\mathrm{g}} \sqrt{2 \pi}} \exp \left[-0.5\left(\frac{\log D-\log D_{\mathrm{g}}}{\log \sigma_{\mathrm{g}}}\right)^{2}\right] .
$$

\section{EFFEGTS OF SNOW PARAMETERS ON EXTINC- TION PROPERTIES}

An attractive approach to modeling snow in the microwave spectrum assumes that snow can be described as discrete scatterers. The discrete-scatter model characterizes the ice particles as spheres with radius $a$, permittivity $\epsilon_{8}$ and fractional volume of scatters $v_{t}$, or multi-size $a_{\mathrm{j}}$, permittivities $\epsilon_{\mathrm{sj}}$ and fractional volume of scatterers $v_{\mathrm{j}}$, imbedded inside a homogeneous medium with permittivity $\epsilon$. The Rayleigh-scattering theory is used to calculate the volume-scattering coefficient of snow because the ice-particle size is much smaller than the wavelength. Under the near-field consideration, the coherence of random scatterers can be taken into account by introducing an effective propagation constant $K$ and analytically modifying the extinction coefficient and albedo (Ding and Tsang, 1988; Tsang, 1992). This is the so-called dense radiative-transfer theory. The scatter-
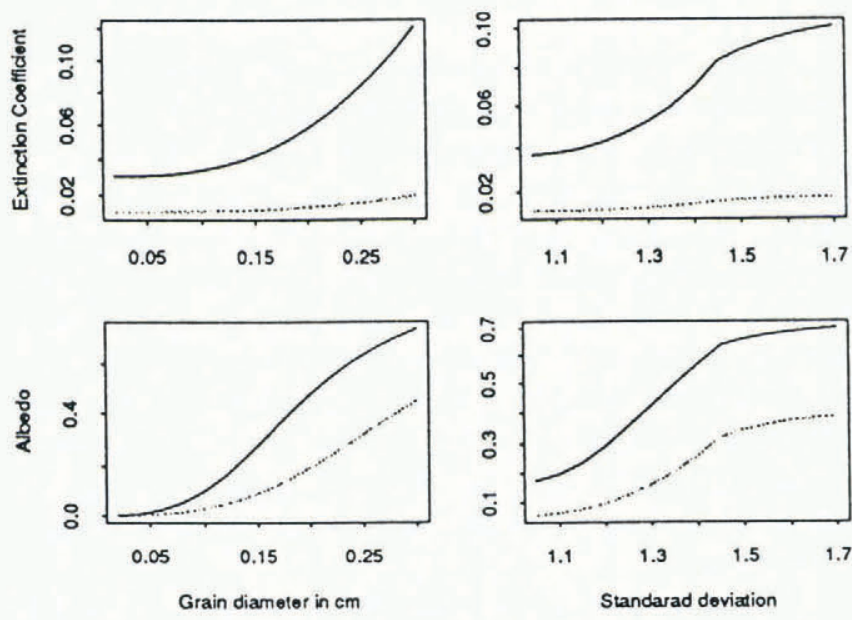

Fig. 1. Comparison of the effect of both particle size and size variation on the extinction properties of dry snow at frequencies of $9.6 \mathrm{GHz}$ (solid line) and $5.3 \mathrm{GHz}$ (dashed line) for a single particle-size system (on the left) and a multi particle-size system with a log-normal distribution function (on the right). The volume fraction of ice and geometric mean of ice particles are 0.32 and $0.12 \mathrm{~cm}$, respectively. 
ing properties of snow under the near-field consideration are calculated by the quasicrystalline approximation with coherent potential using the Percus-Yevick pair-distribution function.

Figure 1 shows the comparison of the effect of both particle size and size variation on the extinction properties of dry snow at frequencies $9.6 \mathrm{GHz}$ and $5.3 \mathrm{GHz}$ for a single particle-size system (on the left) and a multi particle-size system with a log-normal distribution function (on the right). Ten classes of ice-particle sizes and particle-number densities were sampled from the lognormal distribution function determined by the given snow parameters. Scattering increases significantly with the increase of the microwave frequency and the particle size. This is because the scattering coefficient is inversely proportional to the fourth power of the wavelength and is directly proportional to the third power of the particle radius for a given snow density. Since the absorption coefficient is only proportional to the inverse of the wavelength, increases in the scattering coefficient are much greater than the absorption coefficient for a given snow density and particle size when increasing frequency. For small particles (diameters less than $0.5 \mathrm{~mm}$ ), the wave interaction is dominated by absorption. When the particle size increases, scattering increases significantly. This suggests that particle-size variation has a great impact on the determination of the scattering properties of dry snowpacks even when there are few large ice particles in a given layer.

For a given geometric mean of particle size and snow density, increasing the particle-size variation results in a decrease of the maximum height of the particle-size distribution curve. It also changes both the range of particle sizes and volume fractions occupied by each class interval of particle size. As long as the particles follow a log-normal distribution, an increase in the size range increases the volume fraction of particle-size classes that are greater than the geometric mean. Because the volume-scattering coefficient is proportional to the third-power of the particle radius, the large-size particles play a very important role in the extinction properties of

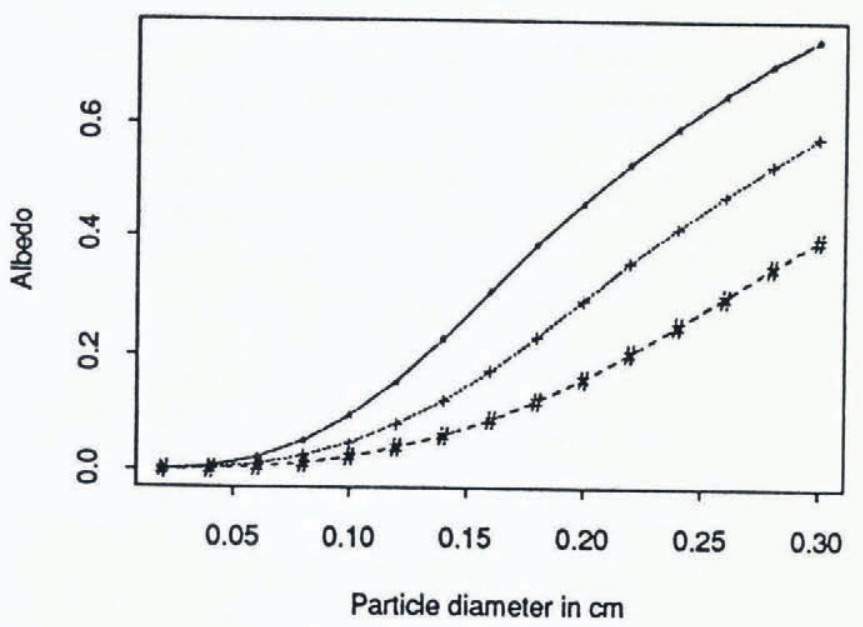

Fig. 2. Effect of snow density on the volume-scattering albedo at a frequency of $5.3 \mathrm{GHz}$. The volume fractions of ice are ${ }^{*}-0.15$; + -0.25; \#-0.35. snowpacks. Therefore, a significant increase in both extinction coefficient and albedo is observed when greater particle-size ranges are present.

For a given frequency, the volume-absorption coefficient is proportional to the volume fraction of ice particles. Figure 2 shows the effect of snow density on the extinction properties at a frequency of $5.3 \mathrm{~Hz}$. The sensitivities of scattering interaction to the particle size at different snow densities have a peak value at which the volume fraction of ice particles is about 0.15 . In the case of a small volume fraction of scatterers, such as clouds, the independentscattering assumption is still valid in the microwave region because the particles are spatially distributed far from each other. Ice particles in natural snowpacks are generally considered as densely distributed scatterers, which means the distances between the particles are much smaller than the wavelength. The near-field effect causes greater coherent scattering from ice particles at higher densities. For a given particle size, increasing snow density increases the number of scatterers per unit volume. But the increasing rate of absorption is much greater than the rate of scattering because the near-field effect on scattering also increases. As a net result, the volume-scattering albedo and the sensitivity of the scattering properties to particle size are negatively related to snow density.

\section{SELECTING THE REPRESENTATIVE GRAIN- SIZE}

It is a long-standing problem to find an appropriate grain-size measurement for the purpose of relating snow physical properties to its microwave-extinction properties. For microwave modeling and interpreting radar backscattering signatures, we are interested in the effect of iceparticle size on the extinction properties of snowpacks, and we would like to express this by specifying a representative particle size. The optically equivalent particle size is defined as a particle size at which the extinction properties from a natural snow volume is equal to that obtained from an ideal snow volume of a uniformly distributed collection of ice particles with a single size. Generally, natural snowpacks show a particle-size distribution at a given snow depth which varies along the vertical profile. In Rayleigh scattering, the determination of the particle size is not important for calculating the volume-absorption coefficient for dry snow because it is only related to the total volume fraction of ice particles. On the other hand, knowledge of the particle size is very important in determining the volume-scattering coefficient for dry snow. We represent the optically equivalent particle size as a mean size, which is weighted with respect to the scattering properties for a natural snowpack. Mathematically, we can write this idea as

$$
\begin{gathered}
\frac{2|K|^{4} v_{\mathrm{t}}|\hat{y}|^{2} \bar{a}^{3}\left(1-v_{\mathrm{t}}\right)^{4}}{\kappa_{\mathrm{e}}|D|^{2}\left(1+2 v_{\mathrm{t}}\right)^{2}}=\frac{2|K|^{4}}{\kappa_{\mathrm{e}}|D|^{2}} \sum_{s_{\ell}=1}^{L} v_{s_{\ell}} \hat{y}_{s_{\ell}} \\
\left\{a_{s_{\ell}}^{3} \hat{y}_{s_{j}}^{*}+\sum_{s_{j}=1}^{L} 8 \pi^{3} a_{s_{j}}^{3} n_{s_{j}} \hat{y}_{s_{j}}^{*} H_{s_{j}} s_{\ell}(p=0)\right\} .
\end{gathered}
$$


The left side of the equation represents the volumescattering albedo for a single-size, single-species particle system. The right side of the equation is the volumescattering albedo for a particle system with a distribution of particle sizes. $\bar{a}$ is the optically equivalent particle size, $\kappa_{\mathrm{e}}$ is the volume-extinction coefficient, $K$ is the effective wave number in the snowpack. $v_{s_{\ell}}$ is the fraction of species $s$ of size $\ell, v_{\mathrm{t}}$ is total volume fraction of ice particles, taken as the sum of the volume fractions of the size classes, and $n_{s_{j}}$ is the number density of the species in a size class. In addition

$$
\begin{gathered}
D=1-\sum_{s_{\ell}=1}^{L} v_{s_{\ell}} \hat{y}_{s_{\ell}} \\
\hat{y}_{s_{j}}=\frac{k_{s_{j}}-k^{2}}{3 K^{2}+\left(k_{s_{j}}^{2}-k^{2}\right)} .
\end{gathered}
$$

Here $k_{s_{j}}$ is the wave number in ice, the only species, and $H_{s_{j} s_{\ell}}(p)$ is the structure factor, which is the Fourier transform of the pair-distribution function $g_{s_{j} s_{\ell}(r)}$. This is defined by

$$
H_{s_{j} s_{\ell}}(p)=\frac{1}{(2 \pi)^{3}} \int \mathrm{d} r\left[g_{s_{j} s_{\ell}}(r)-1\right] \exp (-i p r) .
$$

We consider the permittivity of all particles in dry snow to be the same, so this relation can be further simplified to

$$
\begin{aligned}
\bar{a}^{3}= & \frac{\left(1+2 v_{\mathrm{t}}\right)^{2}}{v_{\mathrm{t}}\left(1-v_{\mathrm{t}}\right)^{4}} \sum_{s_{\ell}=1}^{L} v_{s_{\ell}} \\
& {\left[a_{s_{\ell}}^{3}+\sum_{s_{j}=1}^{L} 8 \pi^{3} a_{s_{j}}^{3} n_{s_{j}} H_{s_{j} s_{\ell}}(p=0)\right] . }
\end{aligned}
$$

This measurement of the particle size can be considered as an equivalent particle size for characterizing a dry snowpack with a particle-size distribution.

Figure 3 shows that the optically equivalent iceparticle size is nearly proportional to the standard deviation of the ice-particle sizes and the ice-volume fraction, as well as the geometric diameter. The optically

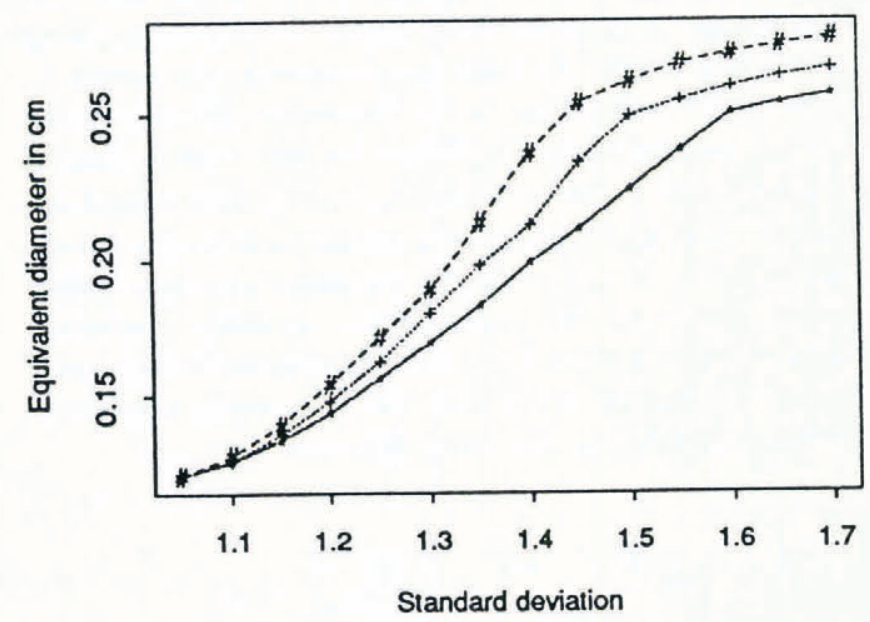

Fig. 3. The calculated optically equivalent ice-particle size. The geometric mean of ice particles is $0.12 \mathrm{~cm}$. The volume fractions of ice are ${ }^{*}-0.15 ;+-0.25 ; \#-0.35$. equivalent ice-particle size increases as the standard deviation increases for a given geometric mean diameter. A larger ice-particle size is required to produce the same amount of scattering in denser snow because of the near-field effect.

In Equation (14), we show that the optically equivalent particle size is independent on both the frequency and the permittivity of ice as long as Rayleigh scattering can be assumed. It is possible to determine the optically equivalent particle size from measurements only of snowpack parameters without sampling the particlesize distribution and calculating the structure factors. This is because the structure factors $H_{s_{j} s_{\ell}}$ are only functions of snowpack parameters (particle sizes, number densities and volume fractions in each particle-size class).

The log-normal distribution possesses moments of any order. The $m$ th moment about the origin is denoted by $\mu_{m}^{\prime}(D)$, and, in general, can be expressed as

$$
\mu_{m}^{\prime}(D)=\exp \left(m \log D_{\mathrm{g}}+0.5 m^{2} \log ^{2} \sigma_{\mathrm{g}}\right) .
$$

Based on this relation, the statistical ice diameters can be obtained which can be related to definite geometrical properties of ice particles, such as particle volume, surface area and linear dimension. Moreover, the statistical diameters are weighted differently by particle-number densities, by weight or volume, or by particle surface. A convenient general expression for classifying the statistical diameters is

$$
D_{m, n}=\left[\frac{\Sigma N_{i} D_{i}^{m}}{\Sigma N_{i} D_{i}^{n}}\right]^{1 /(m-n)}=\left[\frac{\mu_{m}^{\prime}(D)}{\mu_{n}^{\prime}(D)}\right]^{1 /(m-n)},
$$

where $m$ and $n$ are integers whose values determine the various types of averages. For example, the meanweighted diameter is

$$
D_{4,3}=\frac{\mu_{4}^{\prime}(D)}{\mu_{3}^{\prime}(D)}
$$

considered to be a volume-weighted diameter. The goal is to select a suitable particle-size measurement from which the extinction properties can be characterized. By comparing the optically equivalent particle size obtained by Equation (14) and the measurements of different statistical particle sizes for different snow densities and ice particle-size distribution parameters, we find that the volume-weighted diameter $D_{4,3}$ is directly proportional to the representative particle size for a given snow density. We now need to consider the effect of the ice-volume fraction on determining the optically equivalent particle size $D_{\mathrm{e}}^{\prime}$ because of the near-field effect. The statistical relationship can be written as

$$
D_{\mathrm{e}}^{\prime}=\left(1.2+v_{\mathrm{t}}-2 v_{\mathrm{t}}^{2}\right) D_{4,3} .
$$

This equivalent or representative grain-size accounts for the range of particle sizes in a log-normal size distribution and the near-field effect in dense media, such as a snowpack.

\section{CONGLUSIONS}

The critical issue in remote sensing of snow physical properties is how to relate actual snow parameters to 
remote-sensing data at the frequencies of interest. This requires accurate measurements of the snow parameters and accurately describing the extinction properties as a function of the snow parameters. Ice particle-size variation has a great impact on interpreting the relations between observed microwave signals and snowpack parameters using discrete-scatterer theory. In this study, we assumed that the ice particle-size distribution in seasonal snow can be characterized as a log-normal distribution function. The required parameters (the geometric mean diameter and standard deviation of particle diameters) for fully describing the particle-size variation and distribution can be directly measured by the stereological variables, the mean of the first, second and third moment intersection lengths from snow sections. The dense-media model requires the size distribution and the structure factor. In the scattering behavior, each size contributes to its own scattering as well as to the interference from its neighbors as weighted by the pair-distribution function. Using the concept of the optically equivalent particle size, we found that for a snowpack with particle-size variations, the optically equivalent particle size can be determined from snowpack parameters - snow density, geometric mean diameter and standard deviation of particle diameters.

\section{REFERENCES}

Colbeck, S. C. 1986. Statistics of coarsening in watersaturated snow. Acta Metall., 34, 347-352.

Davis, R. E., J. Dozier and A. T. C. Chang. 1987. Snow property measurements correlative to microwave emission at $35 \mathrm{GHz}$. IEEE Trans. Geosci. Remote Sensing, GE-25(6), 751-757.
DeHoff, R.T. 1965. The estimation of particle size distribution from simple counting measurements made on random plane sections. Trans. AIME, 233, 975.

Ding, K.H. and L. Tsang. 1988. Effective propagation constants of dense nontenuous media with multispecies of particles. Fournal of Electromagnetic Waves and Applications, 2, 757-777.

Dozier, J. 1989. Remote sensing of snow in visible and near-infrared wavelengths. In Asrar, G., ed. Theory and applications of optical remote sensing. New York, John Wiley, 527-547.

Hüppe, R. 1986. S- and X-band signature measurements of snow. In Mätzler, C., ed. Proceedings of a Workshop on Microwave Signatures of Arctic Sea Ice Under Summer Melt Conditions. Bern, European Association of Remote Sensing Laboratory, 70-81.

Kinosita, S. and G. Wakahama. 1960. Thin sections of deposited snow made by the use of aniline. Contrib. Inst. Low Temp. Sci., Ser. A, 15, 35-45.

Koh, G. 1984. Snow Symposium IV. Approach to snow propagation modeling. CRREL Spec. Rep. 84-35, 247258.

Tsang, L. 1992. Dense media radiative transfer theory for dense discrete random media with particles of multiple sizes and permittivities. In Prion, A., ed. PIER 6 . Progress in Electromagnetic Research. New York, Elsevier, $181-225$.

Underwood, E.E. 1970. Quantitative stereology. Menlo Park, CA, Addison-Wesley Publishing Company.

The accuracy of references in the text and in this list is the responsibility of the authors, to whom queries should be addressed. 